\title{
Overdamped sine-Gordon kink in a thermal bath
}

\author{
Niurka R. Quintero* and Angel Sánchez ${ }^{\dagger}$ \\ Grupo Interdisciplinar de Sistemas Complicados (GISC), Departamento de Matemáticas, Universidad Carlos III de Madrid, \\ Edificio Sabatini, Avenida de la Universidad 30, E-28911 Leganés, Madrid, Spain \\ Franz G. Mertens \\ Physikalisches Institut, Universität Bayreuth, D-95440 Bayreuth, Germany
}

(Received 19 January 1999)

\begin{abstract}
We study sine-Gordon kink diffusion at finite temperature in the overdamped limit. By means of a general perturbative approach, we calculate the first- and second-order (in temperature) contributions to the diffusion coefficient. We compare our analytical predictions with numerical simulations. The good agreement allows us to conclude that, up to temperatures where kink-antikink nucleation processes cannot be neglected, a diffusion constant linear and quadratic in temperature gives a very accurate description of the diffusive motion of the kink. The quadratic temperature dependence is shown to stem from the interaction with the phonons. In addition, we calculate and compute the average value $\langle\phi(x, t)\rangle$ of the wave function as a function of time, and show that its width grows with $\sqrt{t}$. We discuss the interpretation of this finding and show that it arises from the dispersion of the kink center positions of individual realizations which all keep their width.
\end{abstract}

[S1063-651X(99)08407-X]

PACS number(s): 05.40.-a, 41.20.Jb, 74.50.+r, 85.25.Cp

\section{INTRODUCTION}

There is no longer any controversy about the physical relevance of noise effects in spatially extended, nonlinear systems $[1,2]$ : Indeed, the pervasive, joint role of nonlinearity and (static or dynamic) disorder has already been recognized in biophysics, electronics, optics, fluids, condensed matter, computational physics, etc. In most of these fields, nonlinear phenomena involve nonlinear coherent excitations, such as solitons or solitary waves, which play a key part in the corresponding system dynamics. It is because of this nowadays well established fact that much effort has been devoted to understanding how stochastic perturbations affect solitons, mostly during the decade of the 1980s (see Refs. [3-5] for reviews). In fact, early numerical simulations [6] already revealed that $\phi^{4}$ solitary waves underwent Brownian-like motion in the presence of additive white noise, i.e., of thermal fluctuations. Subsequent works focused on the study of soliton diffusion, since it may be crucial in a number of problems, such as photoexcitation dynamics, photoconductivity of conducting polymers, or transport by phase solitons in charge-density-wave systems, to name a few [7].

Among the different soliton-bearing nonlinear models which have been studied in the above context, one which has received a great deal of attention is the sine-Gordon $(\mathrm{sG})$ equation. The interest in this model is both theoretical, as it displays the main features of more realistic and complicated cases while remaining analytically tractable, and applied, as it very approximately describes the dynamics of many physically relevant systems, such as one-dimensional magnets [8] or long Josephson junctions [9], for instance. Soliton diffu-

\footnotetext{
*Electronic address: kinter@math.uc3m.es

†Electronic address: anxo@math.uc3m.es

¥Electronic address: franz mertens@theo.phy.uni-bayreuth.de
}

sion governed by the sG (and other nonlinear Klein-Gordon equations) has been studied along two main, different lines which are discussed and compared, e.g., in Ref. [10]. The first one consists of considering extended excitations of the system (phonons) in equilibrium with both a single sG soliton and a heat bath at temperature $T$. This approach leads to two distinct diffusion regimes: anomalous diffusion, characterized by a diffusion constant proportional to $T^{2}$, and viscous diffusion, when the appearance of a dynamical damping coefficient yields a diffusion constant proportional to $T^{-1}$. We will not follow this approach here; the interested reader is referred to the detailed review by Wada [11]. The second manner is $\grave{a} l a$ Langevin, i.e., introducing the influence of an external thermal bath by means of local fluctuations of the string and a local damping force related to that by an appropriate fluctuation-dissipation relationship. The corresponding equation of motion is then

$$
\phi_{t t}-\phi_{x x}+\sin (\phi)=-\alpha \phi_{t}+\eta(x, t),
$$

with

$$
\begin{gathered}
\langle\eta(x, t)\rangle=0, \\
\left\langle\eta(x, t) \eta\left(x^{\prime}, t^{\prime}\right)\right\rangle=D \delta\left(x-x^{\prime}\right) \delta\left(t-t^{\prime}\right),
\end{gathered}
$$

where the diffusion coefficient $D=2 \alpha k_{b} T, k_{b}$ being the Boltzmann constant, and $-\alpha \phi_{t}$ being the damping term with a dissipation coefficient $\alpha$. This equation has been considered a number of times in the literature (see, e.g., Ref. [3] and references therein; see also Ref. [12] for related experimental work).

In this work, we focus on the Langevin version of the problem, with the aim of improving the analytical results obtained in the aforementioned works as well as of verifying them by numerical simulations specifically planned to that 
end. Furthermore, we concern ourselves with the overdamped limit of the $\mathrm{sG}$ equation, which reads

$$
\alpha \phi_{t}-\phi_{x x}+\sin (\phi)=\epsilon \eta(x, t, \phi, \ldots) .
$$

Note that we have introduced a factor $\epsilon$ in front of the noise term for convenience in the analytical calculations in Sec. II. This equation (without noise, $\epsilon=0$ ) was already considered by Eilenberger in Ref. [13], as the limit of the sG equation (1) in the case when the dissipation effect is strong enough in Eq. (1), and there is an input of energy into the system (see, e.g., Refs. $[14,15]$ and references therein). On the other hand, Eq. (3), with additive noise as in Eq. (2), is interesting in itself: For example, it has been proposed as a model for crystal growth (see Refs. [16-18] and references therein). Equation (3) has also been studied to analyze the kink contribution to transport properties when the system is driven and thermally activated [16,19-21]. In particular, the work of Kaup [21] is the most closely related to the present one, as it presents a singular perturbation theory to compute the firstorder (in $T$ ) correction to the kink mobility as well as the change of its shape. However, to our knowledge the free diffusion problem for the overdamped $\mathrm{sG}$ equation has not been adressed in the literature to date and, therefore, we believe that our results will be interesting by themselves. On the other hand, we also hope that what we learn in this case can be used toward obtaining a more complete, accurate picture of the full sG problem; we will discuss this question in the conclusions.

The outline of the paper is as follows: In Sec. II, using a general perturbative method [13] which we recall in detail, we calculate the correlation functions of the position and the velocity of the kink center up to second order in $k_{b} T$, as well as the diffusion coefficient and the mean value $\langle\phi(x, t)\rangle$ for fixed $t$. In Sec. III we numerically integrate the stochastic partial differential equation (3), with noise given by Eq. (2), using the Heun scheme [22], and compute the time correlation function of the position of the kink center and the diffusion coefficient. We compare these results with the theoretical ones obtained in Sec. II and find an excellent agreement. Finally, in Sec. IV we discuss our results, summarize our main conclusions, and sketch lines for future research.

\section{A GENERAL PERTURBATIVE APPROACH}

Following the ansatz proposed in Refs. [13,23], we assume that the solution of Eq. (3) can be expanded as

$$
\phi(x, t)=\phi_{0}[x-X(t)]+\int_{-\infty}^{+\infty} d k A_{k}(t) f_{k}[x-X(t)]
$$

where $f_{k}[x-X(t)]$ are the eigenfunctions of the linearized version of Eq. (3) [with $\epsilon=0]$, which along with $f_{T}[x$ $-X(t)]=\left(\partial \phi_{0} / \partial x\right)[x-X(t)]$, form a complete set of orthogonal eigenfunctions (see the Appendix). The first term in expansion (4) represents the translational mode related to the position of the kink center $X(t)$, whereas the second one characterizes the phonon modes (linear excitations around a kink) of the system. We will focus on the kink center motion as described by $X(t)$, as it is well established that such a particlelike picture is very generally enough to describe the behavior of the kink as a whole ( $X$ playing the role of a collective coordinate; see, e.g., Ref. [24] for a review).

In order to calculate the dynamics of the kink center, we begin by inserting Eq. (4) into Eq. (3), and projecting on the orthogonal basis [see the Appendix, relationships (A7)] we obtain a system of differential equations for the unknown functions $X(t)$ and $A_{k}(t)$ :

$$
\begin{aligned}
\dot{X}(t)= & -\frac{1}{8} \dot{X}(t) \int_{-\infty}^{+\infty} d k A_{k}(t) I_{1}(k)-\frac{1}{16 \alpha} \int_{-\infty}^{+\infty} d k \int_{-\infty}^{+\infty} d k^{\prime} A_{k}(t) A_{k^{\prime}}(t) R_{3}\left(k, k^{\prime}\right) \\
& +\frac{\sqrt{D}}{8 \alpha} \int_{-\infty}^{+\infty} f_{T}[x-X(t)] \eta(x, t) d x-\frac{1}{48 \alpha} \int_{-\infty}^{+\infty} d k \int_{-\infty}^{+\infty} d k_{1} \int_{-\infty}^{+\infty} d k_{2} A_{k}(t) A_{k_{1}}(t) A_{k_{2}}(t) R_{6}\left(k, k_{1}, k_{2}\right), \\
\frac{\partial A_{k}}{\partial t}+\frac{\omega_{k}^{2}}{\alpha} A_{k}(t)= & \dot{X}(t) \int_{-\infty}^{+\infty} d k A_{k}(t) I_{3}\left(k, k^{\prime}\right)+\frac{1}{2 \alpha} \int_{-\infty}^{+\infty} d k \int_{-\infty}^{+\infty} d k^{\prime} A_{k}(t) A_{k^{\prime}}(t) R_{4}\left(k, k^{\prime}\right) \\
& -\frac{\sqrt{D}}{\alpha} \int_{-\infty}^{+\infty} f_{k^{\prime}}^{*}[x-X(t)] \eta(x, t) d x+\frac{1}{6 \alpha} \int_{-\infty}^{+\infty} d k \int_{-\infty}^{+\infty} d k_{1} \int_{-\infty}^{+\infty} d k_{2} A_{k}(t) A_{k_{1}}(t) A_{k_{2}}(t) R_{7}\left(k, k_{1}, k_{2}\right),
\end{aligned}
$$

where

$$
\begin{gathered}
I_{1}(k)=\int_{-\infty}^{+\infty} \frac{\partial f_{k}}{\partial \theta} f_{T}(\theta) d \theta=\frac{i \pi \omega_{k}}{\sqrt{2 \pi} \cosh \left(\frac{\pi k}{2}\right)}, \\
R_{3}\left(k, k^{\prime}\right)=\int_{-\infty}^{+\infty} f_{T}(\theta) \frac{\partial f_{T}}{\partial \theta} f_{k}(\theta) f_{k^{\prime}}^{*}(\theta) d \theta=-\frac{i\left(\omega_{k}^{2}-\omega_{k^{\prime}}^{2}\right)^{2}}{4 \omega_{k} \omega_{k^{\prime}} \sinh \left(\frac{\pi \Delta k}{2}\right)}, \quad \Delta k=k^{\prime}-k,
\end{gathered}
$$




$$
\begin{gathered}
I_{3}\left(k, k^{\prime}\right)=\int_{-\infty}^{+\infty} \frac{\partial f_{k}}{\partial \theta} f_{k^{\prime}}^{*}(\theta) d \theta \\
R_{4}\left(k, k^{\prime}\right)=\int_{-\infty}^{+\infty}\left[f_{k^{\prime}}^{*}(\theta)\right]^{2} \frac{\partial f_{T}}{\partial \theta} f_{k}(\theta) d \theta, \quad R_{4}(k, k)=\frac{3 i \omega_{k}}{8 \sqrt{2 \pi} \cosh \left(\frac{\pi k}{2}\right)}, \\
R_{6}\left(k, k_{1}, k_{2}\right)=\int_{-\infty}^{+\infty} \frac{\partial^{2} f_{T}}{\partial \theta^{2}} f_{k}(\theta) f_{k_{1}}^{*}(\theta) f_{k_{2}}(\theta) d \theta \\
R_{7}\left(k, k_{1}, k_{2}\right)=\int_{-\infty}^{+\infty} \cos \left(\phi_{0}\right) f_{k^{\prime}}^{*}(\theta) f_{k}(\theta) f_{k_{1}}^{*}(\theta) f_{k_{2}}(\theta) d \theta .
\end{gathered}
$$

We now recall that, if we set $\epsilon=0$ in Eq. (3), the static kink is an exact solution; hence in what follows we will consider $\epsilon$ as a small perturbative parameter, and expand $A_{k}(t)$ and $X(t)$ in powers of $\epsilon$. By substituting the series $A_{k}(t)$ $=\sum_{n=1}^{\infty} \epsilon^{n} A_{k}^{n}(t)$ and $X(t)=\sum_{n=1}^{\infty} \epsilon^{n} X_{n}(t)$ in Eqs. (5) and (6), we find a set of linear equations for the coefficients of these series. We only write down here the systems of equations up to order $\epsilon^{3}$, leaving out the cumbersome (albeit straightforward) equation for $A_{k}^{3}(t)$. For $O(\epsilon)$,

$$
\begin{gathered}
\dot{X}_{1}(t)=\epsilon_{1}(t), \quad\left\langle\epsilon_{1}(t)\right\rangle=0, \quad\left\langle\epsilon_{1}(t) \epsilon_{1}\left(t^{\prime}\right)\right\rangle=\frac{D}{8 \alpha^{2}} \delta\left(t-t^{\prime}\right), \\
\frac{\partial A_{k}^{1}}{\partial t}(t)+\frac{\omega_{k}^{2}}{\alpha} A_{k}^{1}(t)=\frac{\epsilon_{k}(t)}{\alpha}, \quad\left\langle\epsilon_{k}(t)\right\rangle=0, \quad\left\langle\epsilon_{k}(t) \epsilon_{k^{\prime}}\left(t^{\prime}\right)\right\rangle=\frac{D}{\alpha^{2}} \delta\left(t-t^{\prime}\right) \delta\left(k-k^{\prime}\right) .
\end{gathered}
$$

For $O\left(\epsilon^{2}\right)$

$$
\begin{gathered}
\dot{X}_{2}(t)=-\frac{\dot{X}_{1}(t)}{8} \int_{-\infty}^{+\infty} d k A_{k}^{1}(t) I_{1}(k)-\frac{1}{16 \alpha} \int_{-\infty}^{+\infty} d k \int_{-\infty}^{+\infty} d k^{\prime} A_{k}^{1}(t) A_{k^{\prime}}^{1}(t) R_{3}\left(k, k^{\prime}\right), \\
\frac{\partial A_{k}^{2}}{\partial t}(t)+\frac{\omega_{k}^{2}}{\alpha} A_{k}^{2}(t)=\dot{X}_{1}(t) \int_{-\infty}^{+\infty} d k A_{k}^{1}(t) I_{3}(k, k)+\frac{1}{2 \alpha} \int_{-\infty}^{+\infty} d k \int_{-\infty}^{+\infty} d k^{\prime} A_{k}^{1}(t) A_{k^{\prime}}^{1}(t) R_{4}\left(k, k^{\prime}\right) .
\end{gathered}
$$

For $O\left(\epsilon^{3}\right)$

$$
\begin{aligned}
\dot{X}_{3}(t)= & -\frac{\dot{X}_{1}(t)}{8} \int_{-\infty}^{+\infty} d k A_{k}^{2}(t) I_{1}(k)-\frac{\dot{X}_{2}(t)}{8} \int_{-\infty}^{+\infty} d k A_{k}^{1}(t) I_{1}(k)-\frac{1}{16 \alpha} \int_{-\infty}^{+\infty} d k \int_{-\infty}^{+\infty} d k^{\prime} A_{k}^{2}(t) A_{k^{\prime}}^{1}(t) R_{3}\left(k, k^{\prime}\right) \\
& -\frac{1}{16 \alpha} \int_{-\infty}^{+\infty} d k \int_{-\infty}^{+\infty} d k^{\prime} A_{k}^{1}(t) A_{k^{\prime}}^{2}(t) R_{3}\left(k, k^{\prime}\right)-\frac{1}{48 \alpha} \int_{-\infty}^{+\infty} d k \int_{-\infty}^{+\infty} d k_{1} \int_{-\infty}^{+\infty} d k_{2} A_{k}^{1}(t) A_{k_{1}}^{1}(t) A_{k_{2}}^{1}(t) R_{6}\left(k, k_{1}, k_{2}\right) .
\end{aligned}
$$

We now proceed with the first-order equations. The solutions of Eqs. (8a) and (8b) can be written as

$$
X_{1}(t)=\int_{0}^{t} \epsilon_{1}(\tau) d \tau, \quad A_{k}^{1}(t)=\exp \left(-\frac{\omega_{k}^{2} t}{\alpha}\right) \int_{0}^{t} \exp \left(\frac{\omega_{k}^{2} \tau}{\alpha}\right) \epsilon_{k}(\tau) d \tau
$$

respectively. From these relations we can immediately compute averages over the quantities of interest, such as

$$
\begin{gathered}
\left\langle X_{1}(t)\right\rangle=0, \quad\left\langle X_{1}(t) X_{1}\left(t^{\prime}\right)\right\rangle=\frac{D}{8 \alpha^{2}} M, \\
\left\langle\dot{X}_{1}(t)\right\rangle=0, \quad\left\langle\dot{X}_{1}(t) \dot{X}_{1}\left(t^{\prime}\right)\right\rangle=\frac{D}{8 \alpha^{2}} \delta\left(t-t^{\prime}\right),
\end{gathered}
$$




$$
\left\langle A_{k}^{1}(t)\right\rangle=0, \quad\left\langle A_{k}^{1}(t) A_{k}^{1}\left(t^{\prime}\right)\right\rangle=\frac{D}{2 \alpha \omega_{k}^{2}}\left[\exp \left(-\frac{\omega_{k}^{2}\left|t^{\prime}-t\right|}{\alpha}\right)-\exp \left(-\frac{\omega_{k}^{2}\left(t+t^{\prime}\right)}{\alpha}\right)\right],
$$

where $M=\min \left(t, t^{\prime}\right)$. For the next orders, the calculations are more involved but not difficult. After some tedious algebra, from Eqs. (9a)-(10) we find the average values of the position and velocity of the kink center:

$$
\begin{aligned}
& \left\langle X_{2}(t)\right\rangle=0, \quad\left\langle\dot{X}_{2}(t)\right\rangle=0, \\
& \left\langle X_{3}(t)\right\rangle=0, \quad\left\langle\dot{X}_{3}(t)\right\rangle=0,
\end{aligned}
$$

whereas it can be shown that, for large enough times,

$$
\begin{gathered}
\left\langle\left|A_{k}^{2}(t)\right|\right\rangle \sim \frac{3 \sigma k_{b} T}{16 \sqrt{2 \pi} \omega_{k}^{2}}, \\
\sigma=\int_{-\infty}^{+\infty} \frac{d k}{\omega_{k} \cosh \left(\frac{\pi k}{2}\right)} \approx 1.62386 .
\end{gathered}
$$

The corresponding correlation functions for $X_{2}(t)$ and $\dot{X}_{2}(t)$ are

$$
\begin{gathered}
\left\langle X_{2}(t) X_{2}\left(t^{\prime}\right)\right\rangle=\frac{D^{2} M}{512 \alpha^{3}}+\frac{D^{2} \pi}{4096 \alpha^{2}} \int_{-\infty}^{+\infty} \frac{\left[\exp \left(-2 \omega_{k}^{2} M / \alpha\right)-1\right] d k}{\omega_{k}^{2} \cosh ^{2}\left(\frac{\pi k}{2}\right)}, \\
\left\langle\dot{X}_{2}(t) \dot{X}_{2}\left(t^{\prime}\right)\right\rangle=\left\langle\dot{X}_{1}(t) \dot{X}_{1}\left(t^{\prime}\right)\right\rangle \frac{D \pi}{256 \alpha} \int_{-\infty}^{+\infty} \frac{\exp \left(-\omega_{k}^{2}\left|t^{\prime}-t\right| / \alpha\right)-\exp \left(-\omega_{k}^{2}\left(t^{\prime}+t\right) / \alpha\right) d k}{\cosh ^{2}\left(\frac{\pi k}{2}\right)} .
\end{gathered}
$$

Notice that the cross correlation function of $X_{1}(t)$ and $X_{3}(t)$ is of the same order as $\left\langle X_{2}(t) X_{2}\left(t^{\prime}\right)\right\rangle$, and also that $\left\langle X_{1}(t) X_{2}\left(t^{\prime}\right)\right\rangle=0$. So, from Eqs. (8a) and (10), we have

$$
\begin{aligned}
&\left\langle X_{3}(t) X_{1}\left(t^{\prime}\right)\right\rangle=\left\langle X_{2}(t) X_{2}\left(t^{\prime}\right)\right\rangle-\frac{D^{2}}{256 \alpha^{3}} \int_{-\infty}^{+\infty} d k I_{1}(k)\left\{\left(\int_{-\infty}^{+\infty} d m \frac{R_{4}(m, m)}{\omega_{m}^{2}}\right)\left[\frac{M}{\omega_{k}^{2}}+\frac{\alpha\left(\exp \left(-\omega_{k}^{2} M / \alpha\right)-1\right)}{\omega_{k}^{4}}\right]\right. \\
&\left.-\int_{-\infty}^{+\infty} d n \frac{R_{4}(n, n)}{\omega_{n}^{2}} \frac{\alpha}{2 \omega_{n}^{2}-\omega_{k}^{2}}\left[\frac{\left(\exp \left(-2 \omega_{n}^{2} M / \alpha\right)-1\right)}{2 \omega_{n}^{2}}-\frac{\left(\exp \left(-\omega_{k}^{2} M / \alpha\right)-1\right)}{\omega_{k}^{2}}\right]\right\}, \\
&\left\langle\dot{X}_{3}(t) \dot{X}_{1}\left(t^{\prime}\right)\right\rangle=-\frac{1}{8}\left\langle\dot{X}_{1}(t) \dot{X}_{1}\left(t^{\prime}\right)\right\rangle \int_{-\infty}^{+\infty} d k\left\{\left\langle A_{k}^{2}(t)\right\rangle I_{1}(k)-\frac{1}{8}\left\langle\left[A_{k}^{1}(t)\right]^{2}\right\rangle\left|I_{1}(k)\right|^{2}\right\} .
\end{aligned}
$$

Finally, from Eqs. (13), (19), and (21) we obtain the final result, namely, that for large $t$ [i.e., taking the limit $t \rightarrow \infty$ in Eqs. (19) and (21) in all terms except those related to $\left.X_{1}(t)\right]$ the correlation function $\left\langle\dot{X}(t) \dot{X}\left(t^{\prime}\right)\right\rangle$ is given up to order $\epsilon^{4}$ by

$$
\begin{aligned}
\left\langle\dot{X}(t) \dot{X}\left(t^{\prime}\right)\right\rangle & =\epsilon^{2}\left\langle\dot{X}_{1}(t) \dot{X}_{1}\left(t^{\prime}\right)\right\rangle+\epsilon^{4}\left(\left\langle\dot{X}_{2}(t) \dot{X}_{2}\left(t^{\prime}\right)\right\rangle+\left\langle\dot{X}_{1}(t) \dot{X}_{3}\left(t^{\prime}\right)\right\rangle+\left\langle\dot{X}_{3}(t) \dot{X}_{1}\left(t^{\prime}\right)\right\rangle\right)+\cdots \\
& =\frac{\epsilon^{2}}{8}\left\langle\dot{X}_{1}(t) \dot{X}_{1}\left(t^{\prime}\right)\right\rangle\left\{1+\epsilon^{2}\left(\frac{3}{32}+\frac{3}{128} \sigma^{2}\right) k_{b} T\right\}+o\left(\epsilon^{4}\right) .
\end{aligned}
$$

We now return to our original equation notation: We set $\epsilon$ equal to 1 and consider $\sqrt{k_{b} T}$ as the small parameter. When $t$ goes to infinity and imposing $\epsilon=1$, from Eqs. (12), (18), and (20) we find

$$
\left\langle[X(t)]^{2}\right\rangle=\frac{k_{b} T}{4 \alpha} t\left\{1+\left(\frac{3}{32}+\frac{3}{128} \sigma^{2}\right) k_{b} T\right\} .
$$

Note that the slope of this function is the kink diffusion 

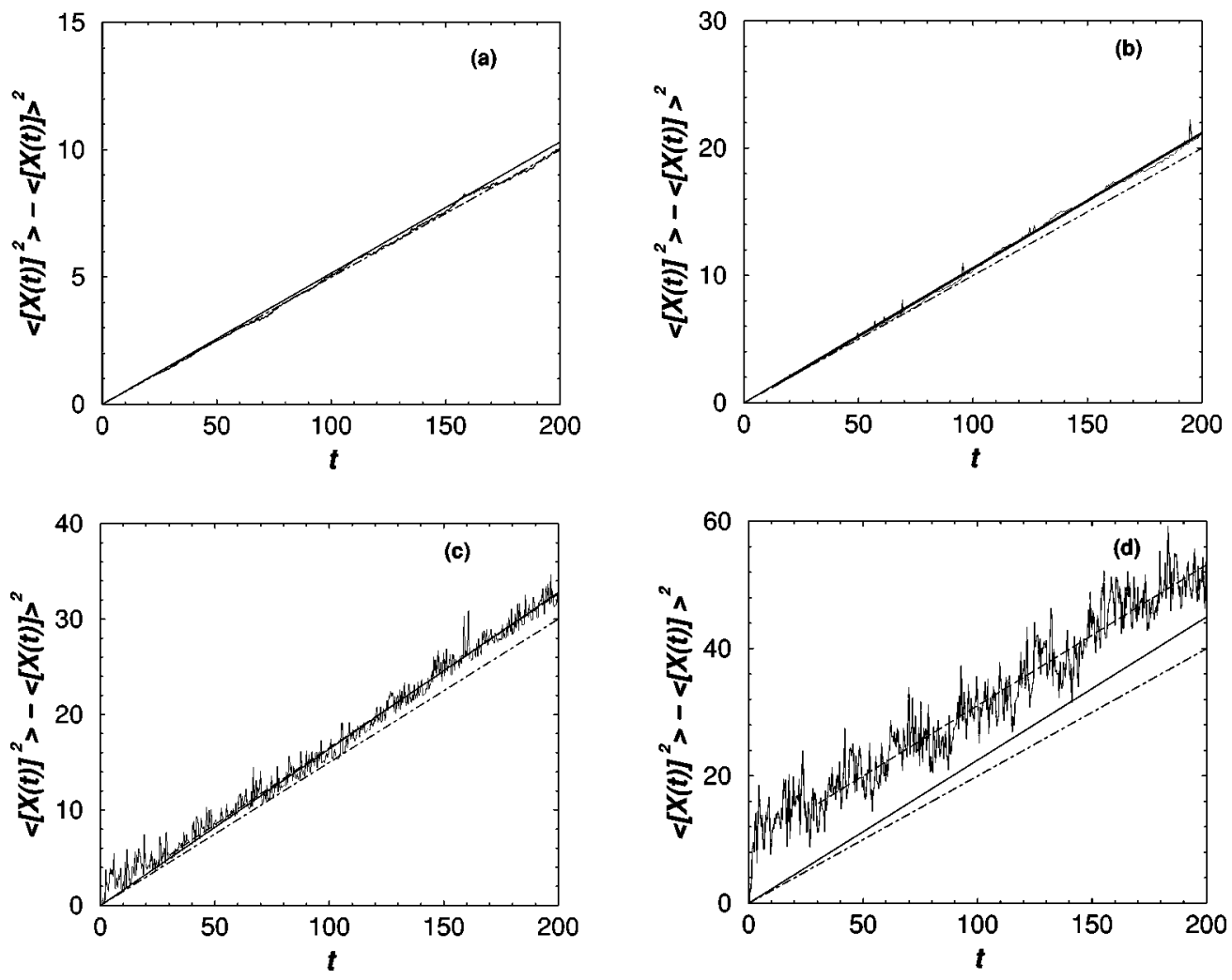

FIG. 1. Simulations with initial condition given by a static kink initially located at $X(0)=0$, and subject to a thermal bath. As a continuous (but wiggly) line, we have plotted $\left\langle[X(t)]^{2}\right\rangle-\langle[X(t)]\rangle^{2}$ as obtained by numerical integration of Eq. (3) for (a) $k_{b} T=0.2$, (b) $k_{b} T=0.4$, (c) $k_{b} T=0.6$, and (d) $k_{b} T=0.8$. Superimposed on these lines, the linear regression of the numerical results for $t \geqslant 30$ is shown (long-dashed line). The solid line is the theoretical prediction $\left\langle[X(t)]^{2}\right\rangle-\langle[X(t)]\rangle^{2}$ from Eq. (23); this line practically overlaps with the linear regression in (a), (b), and (c). The first-order result $\left\langle[X(t)]^{2}\right\rangle-\langle[X(t)]\rangle^{2}$ from Eq. (12) is shown as a dot-dashed line.

coefficient, so if one takes into account the second-order correction one obtains that the diffusion coefficient is a quadratic function of the temperature. We postpone our comments to Sec. IV, where a comparison with the previously available results will be made.

To complete this work, we can calculate in a very simple way the average value of the wave function $\phi(x, t)$ in first order: From Eq. (4), we have that

$$
\langle\phi(x, t)\rangle=\left\langle\phi_{0}\left[x-\epsilon X_{1}(t)\right]\right\rangle+O\left(\epsilon^{2}\right) .
$$

In this last relation we have taken into account that $\left\langle A_{k}(t)\right\rangle$ $=\epsilon\left\langle A_{k}^{1}(t)\right\rangle+O\left(\epsilon^{2}\right)$ and $\left\langle A_{k}^{1}(t)\right\rangle=0$ [see Eq. (14)].

If we solve the corresponding Fokker-Planck equation for $X_{1}$ [see Eq. (8a)], we obtain that the probability distribution function for $X_{1}$ is a Gaussian function given by

$$
p\left(X_{1}\right)=\sqrt{\frac{4 \alpha^{2}}{\pi t D}} \exp \left(-\frac{4 \alpha^{2} X_{1}^{2}}{D t}\right) .
$$

So one can define the average value $\langle\phi(x, t)\rangle$ as

$$
\langle\phi(x, t)\rangle=\int_{-\infty}^{+\infty} d X_{1} p\left(X_{1}\right) \phi_{0}\left[x-\epsilon X_{1}(t)\right] .
$$

Unfortunately we have not found the analytical expression for this integral. But we have calculated it numerically, and below we will compare it to the simulations for the full partial differential equation.

\section{NUMERICAL SIMULATIONS}

For our numerical simulations of the partial differential equation (3), we have used the method of Heun [22], whose stochastic properties are well known and suitable for comparison to our theoretical predictions. We numerically integrate Eqs. (3), with white noise [Eq. (2)], starting from an unperturbed kink at rest and taking the average values over 1000 realizations. The other parameters are $\alpha=1, \Delta x$ $=0.05$, and $\Delta t=0.001$. In the evaluation of the simulations, we have defined the center of the kink as follows: We first find all the lattice points $i$ such that $\phi_{i} \leqslant \pi$ and $\phi_{i+1} \geqslant \pi$, or vice versa. We then interpolate to obtain the points $x_{i}$ where the field $\phi$ crosses $\pi$. In case that, due to the noise-induced deformation of the kink, there is more than one such $x_{i}$, we average them to finally obtain the numerical kink center position, $x_{c}$. As discussed below, this introduces some error, but other alternatives we tested (such as the center of mass, for instance) gave results which did not really represent the kink location, and moreover its calculation from numerics is much less accurate. Once the center is obtained, we also computed its variance $\left\langle[X(t)]^{2}\right\rangle-\langle[X(t)]\rangle^{2}$.

Figures $1(\mathrm{a})-1(\mathrm{~d})$ show a comparison of our numerical results with the analytical predictions [Eqs. (12) and (23)], for different values of $k_{b} T$. We see that there is an excellent agreement between theory and numerics except for the highest value of $k_{b} T$ [Fig. 1(d)]. We have checked that this disagreement arises from the way we compute the kink center: For such large values of the noise, points where $\phi(x, t)=\pi$ 


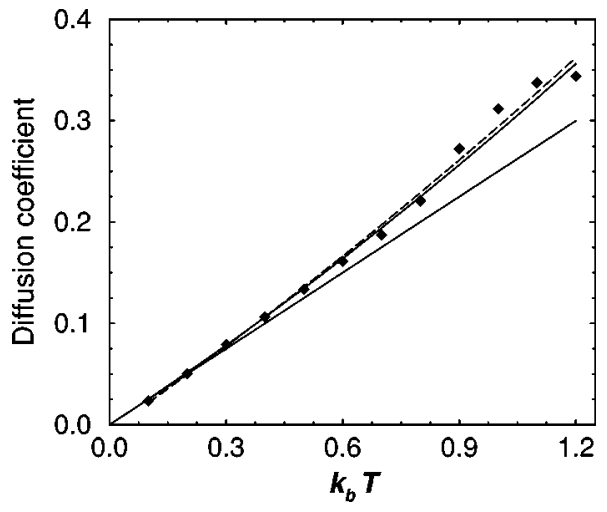

FIG. 2. Lower solid line: the function $D_{1}$; upper solid line: $D_{2}$, which represent the first- and second-order results for the kink diffusion coefficient [see Eqs. (12) and (23)]. Diamonds represent the numerical values of the kink diffusion coefficient, obtained by numerical integration of Eq. (3) with final time $t_{f}=200$ (as in Fig. 1) and different values of $k_{b} T$. A quadratic regression of these numerical values is also plotted (long-dashed line).

are found all over the system, irrespective of their distance to the kink center (we note, however, that the temperature was not high enough to create new kink-antikink pairs). Those points contribute to the center position through our averaging procedure, and in fact their contribution can be shown to be additive, i.e., it amounts to move the whole curve $\left\langle[X(t)]^{2}\right\rangle-\langle[X(t)]\rangle^{2}$ upwards. This is indeed what occurs in Fig. 1(d), and as we will see below the slope is very close to the predicted one. The same behavior is found for higher temperatures insofar as no new kinks are created (not shown). Interestingly, a first conclusion that can be drawn from these figures is that already for not so high temperatures, $k_{b} T \geqslant 0.4$, as time passes the kink behavior becomes more and more different from the first-order prediction, showing clearly the necessity for the second-order correction.

We have calculated the numerical values of the diffusion coefficient for several temperatures by taking the slope of $\left\langle[X(t)]^{2}\right\rangle-\langle[X(t)]\rangle^{2}$, which we obtain from a linear fit of the data for not so early times $(t \geqslant 30)$ to avoid transient effects coming from the adjustment of the kink to the heat bath. Note also that our prediction for the second-order con-

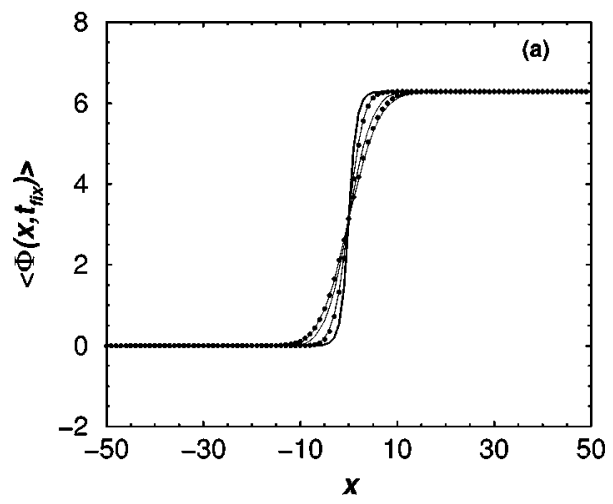

tribution was obtained in the large-time limit, so we should not try to fit the entire evolution. The figures also show those linear regressions. Subsequently, in Fig. 2 we compare the computed slopes with the first- and second-order coefficients $D_{1}=k_{b} T / 4 \alpha$, and $D_{2}=\left(k_{b} T / 4 \alpha\right)\left\{1+\left(\frac{3}{32}+\frac{3}{128} \sigma^{2}\right) k_{b} T\right\}$ [see Eqs. (12) and (23)]. The comparison is once again very good, and points out very clearly that for values of $k_{b} T$ as low as 0.3 , the first-order prediction begins to deviate from the diffusion constant measured in the simulations. In addition, the quadratic fit to the simulation results, shown as a longdashed line in Fig. 2, practically coincides with the secondorder prediction in the whole studied range.

As a final verification of our results, in Fig. 3 we plot the mean value $\langle\phi(x, t)\rangle$ of the wave function at three different times along its evolution, both as obtained from the numerical simulation of the partial differential equation and from the numerical evaluation of Eq. (26). The perfect agreement between these expressions provides us with a hint as to how to derive an approximate analytical estimate of the evolution of $\langle\phi\rangle$ from integral (26). From Fig. 3 one immediately concludes that the width of $\langle\phi\rangle$ increases with temperature and time. Let us define the width of $\langle\phi\rangle$ by

$$
L(t)=\sqrt{\frac{\int_{-\infty}^{+\infty} x^{2}\left\langle\left[\phi_{x}(x, t)\right]^{2}\right\rangle d x}{\int_{-\infty}^{+\infty}\left\langle\left[\phi_{x}(x, t)\right]^{2}\right\rangle d x}} .
$$

With this definition, we can now calculate $\left\langle\left[\phi_{x}(x, t)\right]^{2}\right\rangle$ by using the distribution function of $X_{1}(t)$; this procedure yields

$$
L(t) \approx \sqrt{L_{0}^{2}+\left\langle\left[X_{1}(t)\right]^{2}\right\rangle}
$$

where

$$
L_{0}^{2}=\frac{\int_{-\infty}^{+\infty} d x\left[x^{2} / \cosh ^{2}(x)\right]}{\int_{-\infty}^{+\infty} d x\left[1 / \cosh ^{2}(x)\right]}=0.8225 .
$$

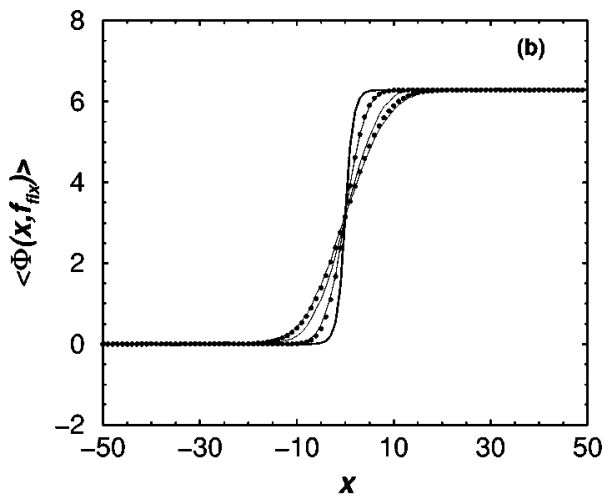

FIG. 3. Solid lines: Snapshots of the evolution of $\langle\phi(x, t)\rangle$, obtained from numerical simulations of the partial differential equation, for fixed times of 40,120, and 200, respectively. The initial kink (unperturbed, at rest) is also included for comparison. The width of $\langle\phi\rangle$ increases as time progresses. The superimposed points have been computed numerically from integral (26). Plots correspond to $k_{b} T=0.4$ (a) and 0.8 (b); the width of $\langle\phi\rangle$ is seen to increase also with temperature. 

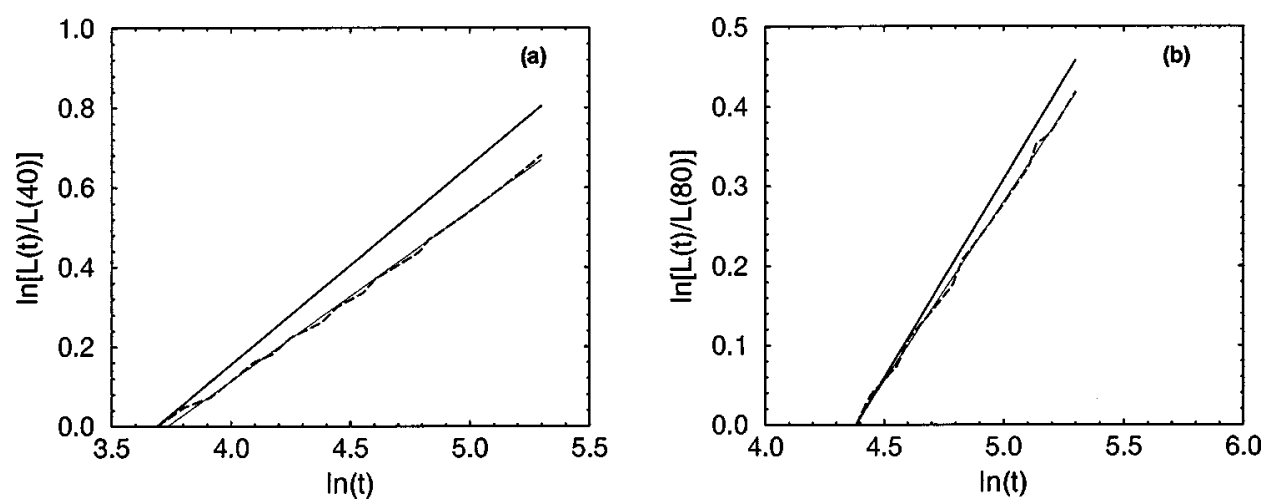

FIG. 4. Solid lines: Analytical values of $\ln \left[L(t) / L\left(t_{\text {fix }}\right)\right]$ for $t_{\text {fix }}=40$ (a) and $t_{\text {fix }}=80$ (b). In both cases, $\alpha=1$ and $k_{b} T=0.6$. Long-dashed lines: numerical values, calculated from Eq. (26). The solid lines over the long-dashed lines correspond to the linear regression of the numerical points.

It is important to note that, of course, we could define $L(t)$ using $\left\langle\phi_{x}(x, t)\right\rangle$ instead of $\left\langle\left[\phi_{x}(x, t)\right]^{2}\right\rangle$ in the above expression, or equivalently another quantity which is localized around the kink center. However, as all possible (and sensible) definitions of $L(t)$ give more or less the same results, the difference between them becomes a constant factor when $\left\langle\left[X_{1}(t)\right]^{2}\right\rangle$ increases above $L_{0}^{2}$ (for example, for large enough $t)$. So we expect that the ratio

$$
\frac{L(t)}{L\left(t_{\text {fix }}\right)} \rightarrow \sqrt{\frac{t}{t_{\text {fix }}}}
$$

for large enough $t$ and $t_{\text {fix }}$.

Figure 4 shows a comparison of this prediction with the numerical evaluation of the width of $\langle\phi\rangle$ from Eq. (26). From these plots we see that the broadening of $\langle\phi\rangle$ indeed behaves as $\sqrt{t}$ : We can compare the analytical slope equal to 0.5 with the numerical ones equal to 0.4276 and 0.4517 for plots (a) and (b), respectively. The slope in (b) is closer to the analytical value due to the fact that $t_{\text {fix }}$ is larger than in case (a), which agrees with the above considerations.

\section{DISCUSSION AND CONCLUSIONS}

As we saw in Sec. III, our second-order theoretical predictions constitute a very accurate description of the kink dynamics for a wide range of temperatures, up to a value of $k_{b} T \simeq 1$. In fact, the range of validity of the analytical results might be somewhat higher, provided a better way to estimate the kink center from the numerical simulations could be devised. In any event, the occurrence of $\pi$ crossings far away from the kink center for values around $k_{b} T \simeq 1$ indicates that further increments of the temperature would undoubtedly produce kink-antikink pairs, thus invalidating our collective coordinate approach which necessarily relies on the identification of the individual kink propagation. We note that this value is a little over $10 \%$ of the kink rest mass $\left(M_{0}=8\right.$ in our units); in this respect, a similar result was obtained in Ref. [25] for the overdamped $\phi^{4}$ model by means of a similar perturbative approach (with the caveat that the numerical data presented in Ref. [25] only allow one to guess what is the range of validity of their results).
It is interesting to pursue further the comparison of the results for the sG and $\phi^{4}$ cases. In our calculations for the $\mathrm{sG}$ equation, we have found that the second-order correction is clearly smaller (albeit relevant) than the first-order one. The structure of the perturbative calculation allows one to identify the origin of that correction: It comes from the interaction of the phonons (described by the functions $A_{k}$ ) with the kink. Now, in the $\phi^{4}$ case, the situation is quite different: Indeed, the second-order correction is much larger than the one we find here, and the reason for this is the so-called internal mode, present for $\phi^{4}$ kinks and absent in the sG case. The coupling between this internal mode (which has been shown to act as a reservoir of energy available for exchange with the kink translation mode [26]) and the kink motion can be shown, by a careful examination of the calculation in Ref. [25], to be responsible for most of the secondorder correction, while the phonons produce a second-order term comparable to the one we have found. We thus see that, while the range of validity of the analytical approach is in principle the same in both cases, the physics is certainly different, and in fact the question arises as to the validity of this kind of perturbative calculation for the $\phi^{4}$ problem in view of the large contribution of the internal mode. This is an interesting question that deserves further analytical and numerical work.

Returning to our results for the sG kink, the fact that the second-order correction is smaller than the first-order term makes us confident that our expansion is likely to be free of problems coming from secular terms. This belief is reinforced by the result that, up to the validity range discussed above and limited by kink-antikink creation phenomena, the second-order result describes the kink behavior very accurately, which deviates very little from the predicted diffusive motion. It is then reasonable to expect higher-order contributions (whose calculation is extremely cumbersome, but feasible in principle) to be negligible, thus yielding our theoretical result as the final one for the kink diffusion in the overdamped sG problem. In this context, it is also important to realize that Eqs. (8a) and (8b), which are only first order, can also be obtained following the McLaughlin and Scott procedure [27] (see also Ref. [24]). However, the advantages of the perturbative scheme we have used are, on the one hand, that we were able to obtain the next order in the ex- 


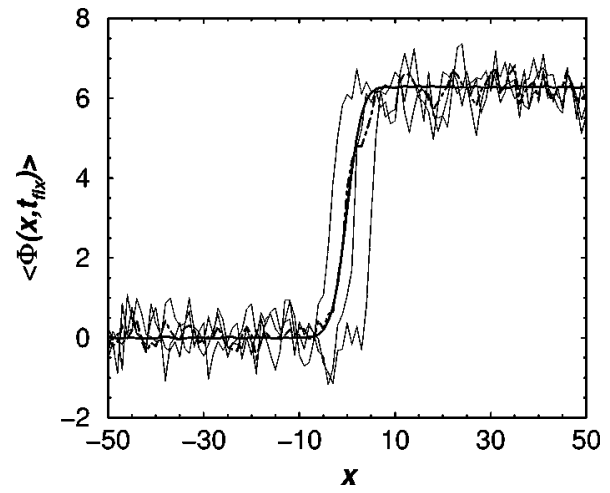

FIG. 5. Average of the wave function for $k_{b} T=0.4$ and $t$ $=200$ obtained from 1000 realizations (wider solid line), compared to the average of only five realizations (dot-dashed line). Also represented are three of these individual realizations. Note the different slope and width of the average values as compared to individual realizations.

pansion, and, on the other hand, that we demonstrated that the second order originates in the interaction between phonon and translational modes of the sG kink.

A final remark on our results relates to the mean value of the wave function $\langle\phi(x, t)\rangle$ as a function of $t$, that must not be interpreted as the shape of the kink, in contrast to the interpretation in Ref. [25]. We first note that the width of the kink cannot increase from its value when unperturbed at rest; the $\mathrm{sG}$ equation, being Lorentz invariant, implies that the kink width diminishes when in motion, and therefore an increasing of the width would be very difficult to understand on physical grounds. Indeed this is not the case. The broadening of the mean wave function in fact comes from the dispersion of the individual realizations, as is immediately seen from Fig. 5. As may be seen, all individual realizations show a width comparable to the initial kink width, which agrees with our physical intuition. The observed $\sqrt{t}$ behavior, discussed at the end of Sec. III, is then evidently related to the fact that the variance of the kink position also has that behavior. The correct interpretation of the width of $\langle\phi(x, t)\rangle$ is that it represents the area in which the kink can be located as its diffusive motion progresses. A similar result was found for multiplicative noise in Ref. [28] (see also Ref. [2] and references therein).

To conclude, we want to stress that our main result is the quadratic dependence of the diffusion constant on the temperature, stemming from the kink-phonon interactions. This has been verified numerically to a high degree of accuracy. We have carried out standard Langevin dynamics simulations following a well grounded procedure, the Heun method, as far as statistical properties are concerned [22]. We can thus be sure that what we are dealing with is indeed the dynamics of a sG kink at finite temperature. Therefore, our analytical calculations and our numerical simulations firmly establish the quadratic dependence of the kink diffusion constant on the temperature. Now the question remains as to the behavior of underdamped sG kinks. Preliminary calculations [29] seem to indicate that for underdamped sG kinks the second-order correction is of the same order as that found here, which would support the applicability of the pre- vious calculations at least for small temperatures and damping that is not too small. To date, to our knowledge, no detailed comparison with numerical simulations has ever been done to check the importance of the second-order correction. On the other hand, it would be interesting to compare the results of our approach with the theoretical analysis and experiments in Ref. [12]. Such a comparison would provide much insight into the importance of second- and higherorder corrections in actual physical systems. Work along these lines is in progress [29].

Note added in proof. After acceptance of this paper, we implemented and improved the algorithm for detecting the kink center in our code. With this new procedure, no spurious contributions (see discussion below Fig. 1) to the variance appear. Specificially, Fig. 1(d) is largely improved, and the numerical results overlap the theoretical prediction, thus confirming the interpretation we have provided of the discrepancy. A detailed report will be given in [29].

\section{ACKNOWLEDGEMENTS}

We are grateful to Esteban Moro, Grant Lythe, and José María Sancho for discussions. Work at GISC (Leganés) was supported by CICyT (Spain) Grant No. MAT95-0325 and DGES (Spain) Grant No. PB96-0119. Travel between Bayreuth and Madrid was supported by "Acciones Integradas Hispano-Alemanas," a joint program of DAAD (Az. 314-AI) and DGES. This research was part of a project supported by NATO Grant No. CRG 971090.

\section{APPENDIX}

One class of solutions of Eq. (3) [with $\epsilon=0$ ] is represented by a static kink

$$
\phi_{0}(x, t)=4 \arctan [\exp (x)]
$$

The perturbations over this equation may be treated by assuming that the solution of Eq. (3) [with $\epsilon=0$ ] has the forms

$$
\phi(x, t)=\phi_{0}(x)+\psi(x, t), \quad \psi(x, t) \ll \phi_{0}(x) .
$$

If we substitute Eq. (A2) into Eq. (3) [with $\epsilon=0$ ], and linearize around $\phi_{0}(x)$, we obtain the following equation for $\psi(x, t)$ :

$$
\alpha \psi_{t}=\psi_{x x}-\left[1-\frac{2}{\cosh ^{2}(x)}\right] \psi
$$

Then, the solution of Eq. (A3) may be written as $\psi(x, t)$ $=f_{k}(x) \exp \left(-\omega_{k}^{2} t / \alpha\right)$, where $f_{k}(x)$ satisfies the eigenvalue problem given by

$$
-\frac{\partial^{2} f_{k}}{\partial x^{2}}+\left[1-\frac{2}{\cosh ^{2}(x)}\right] f_{k}=\omega_{k}^{2} f_{k}
$$


This equation admits the following eigenfunctions with their respective eigenvalues:

$$
\begin{gathered}
f_{T}(x)=\frac{2}{\cosh (x)}, \quad \omega_{T}^{2}=0, \\
f_{k}(x)=\frac{\exp (i k x)[k+i \tanh (x)]}{\sqrt{2 \pi} \omega_{k}}, \quad \omega_{k}^{2}=1+k^{2} .
\end{gathered}
$$

Notice that $f_{T}(x)$ and $f_{k}(x)$ form a complete set of functions with the orthogonality relations

$$
\begin{gathered}
\int_{-\infty}^{+\infty} f_{T}^{2}(x) d x=8, \quad \int_{-\infty}^{+\infty} f_{T}(x) f_{k}(x) d x=0, \\
\int_{-\infty}^{+\infty} f_{k}(x) f_{k^{\prime}}^{*}(x) d x=\delta\left(k-k^{\prime}\right) .
\end{gathered}
$$

[1] Fluctuation Phenomena: Disorder and Nonlinearity, edited by A. R. Bishop, S. Jiménez, and L. Vázquez (World Scientific, Singapore, 1995).

[2] J. García-Ojalvo and J. M. Sancho, Noise in Spatially Extended Systems (Springer, New York, 1999).

[3] F. G. Bass, Yu. S. Kivshar, V. V. Konotop, and Yu. A. Sinitsyn, Phys. Rep. 157, 63 (1988).

[4] A. Sánchez and L. Vázquez, Int. J. Mod. Phys. B 5, 2825 (1991).

[5] V. V. Konotop and L. Vázquez, Nonlinear Random Waves (World Scientific, Singapore, 1994).

[6] T. R. Koehler, A. R. Bishop, J. A. Krumhansl, and J. R. Schrieffer, Solid State Commun. 17, 1515 (1975).

[7] Yu Lu, Solitons and Polarons in Conducting Polymers (World Scientific, Singapore, 1988); G. Gruner, Density Waves in Solids (Addison-Wesley, New York, 1994).

[8] H. J. Mikeska, J. Phys. C 11, 129 (1978).

[9] A. Barone and G. Paternó, Physics and Applications of the Josephson Effect (Wiley, New York, 1982).

[10] B. A. Ivanov and A. Kolezhuk, Phys. Lett. A 146, 190 (1990).

[11] Y. Wada, Prog. Theor. Phys. Suppl. 113, 1 (1993).

[12] M. G. Castellano, G. Torrioli, C. Cosmelli, A. Costantini, F. Chiarello, P. Carelli, G. Rotoli, M. Cirillo, and R. L. Kautz, Phys. Rev. B 54, 15417 (1996).

[13] G. Eilenberger, Z. Phys. B 27, 199 (1977).

[14] Yu. S. Kivshar and B. A. Malomed, Rev. Mod. Phys. 61, 763 (1989).
[15] C. H. Bennett, M. Büttiker, R. Landauer, and H. Thomas, J. Stat. Phys. 24, 419 (1981).

[16] J. Krug and H. Spohn, Europhys. Lett. 8, 219 (1989).

[17] A. Sánchez, D. Cai, N. Grønbech-Jensen, A. R. Bishop, and Z. J. Wang, Phys. Rev. B 51, 14664 (1995).

[18] R. Rangel and L. E. Guerrero, Fractals 3, 533 (1995).

[19] M. Büttiker and R. Landauer, J. Phys. C 13, L325 (1980).

[20] M. Büttiker and R. Landauer, Phys. Rev. A 23, 1397 (1981).

[21] D. J. Kaup, Phys. Rev. B 27, 6787 (1983).

[22] M. San Miguel and R. Toral, in Instabilities and Nonequilibrium Structures $V$, edited by E. Tirapegui and W. Zeller (Kluwer, Dordrecht, 1997).

[23] R. Rajaraman, Solitons and Instantons (North-Holland, Amsterdam, 1982).

[24] A. Sánchez and A. R. Bishop, SIAM (Soc. Ind. Appl. Math.) Rev. 40, 579 (1998).

[25] Jacek Dziarmaga and Wojtek Zakrzewski, Phys. Lett. A 251, 8583 (1999).

[26] D. K. Campbell, J. F. Schonfeld, and C. A. Wingate, Physica D 9, 1 (1983).

[27] D. W. McLaughlin and A. C. Scott, Phys. Rev. A 18, 1652 (1978).

[28] J. Armero, J. M. Sancho, J. Casademunt, A. M. Lacasta, L. Ramírez-Piscina, and F. Sagués, Phys. Rev. Lett. 76, 3045 (1996).

[29] N. R. Quintero, A. Sánchez, and F. Mertens (unpublished). 